\title{
THE ULTRASTRUCTURAL LOCALIZATION OF SEROTONIN IMMUNOREACTIVITY IN MYELINATED AND UNMYELINATED AXONS WITHIN THE MEDIAL FOREBRAIN BUNDLE OF RAT AND MONKEY ${ }^{1}$
}

\author{
EFRAIN AZMITIA ${ }^{2}$ AND PATRICK GANNON
}

Department of Anatomy, Neurobiology Faculty, Mount Sinai School of Medicine, New York, New York 10029

Received November 17, 1982; Revised April 18, 1983; Accepted April 28, 1983

\begin{abstract}
Myelinated and unmyelinated serotonin-containing axons were demonstrated in the medial forebrain bundle of rats and monkeys with an antibody against serotonin $(5-\mathrm{HT})$ conjugated to hemocyanin. The level of 5-HT in the brain was increased by pretreatment of animals with pargyline and $\mathrm{L}$-tryptophan. The addition of nickel salts to the diaminobenzidine-peroxidase reaction mixture increased the intensity of the reaction product. Specific 5-HT immunoreactivity was observed to be localized to membrane-enclosed and membrane-attached compartments.

5-HT immunoreactivity was seen predominantly in unmyelinated axons $(0.2$ to $1.25 \mu \mathrm{m}$ in diameter) surrounded by unlabeled processes. More than half of these 5-HT-immunoreactive fibers were in apposition with unreactive myelinated axons in the hypothalamus of both the rat (55\%) and the monkey (54\%). These appositions may provide the anatomical substrate for the observations that sprouting serotonergic fibers in the adult brain use myelinated axons for contact guidance.

In addition to the unmyelinated fibers, intensely labeled myelinated axons (1.0 to $2.1 \mu \mathrm{m})$ were seen in the medial forebrain bundle of both rats and monkeys. The percentage of 5-HT-immunoreactive myelinated axons appeared greater in the monkey than in the rat $(25.4 \%$ versus $0.7 \%$ of the total number of 5-HT-immunoreactive fibers, respectively). The observation of an increased number of myelinated 5-HT-immunoreactive axons in the monkey hypothalamus as compared to the rat supports the general hypothesis of a trend toward increased myelination during evolution.
\end{abstract}

Serotonin (5-HT)-producing neurons form phylogenetically (Parent, 1981) and ontogenetically (Lauder and Bloom, 1974; Olson and Seiger, 1972) one of the earliest brain-chemical systems. These neurons have highly branched fibers innervating virtually the entire vertebrate forebrain and comprising the most expansive neuronal network yet described in the central nervous system (CNS). Anatomical studies in the adult rat brain have shown that the 5-HT axons ascending from the midbrain raphe nuclei are often seen within known myelinated pathways such as the fornix, cingulum bundle, fasciculus retroflexus, and optic tract (Azmitia and Segal, 1978; Lidov et al., 1980; Steinbusch, 1981). However, other than the observations that the soma of raphe neurons are in direct contact with oligodendrocytes $(\mathrm{Az}-$

\footnotetext{
${ }^{1}$ This work was supported by National Science Foundation Grant BNS 79-06474 and a Career Scientist Award from the I. Hirschl Trust.

${ }^{2}$ To whom correspondence should be addressed, at Department of Biology, New York University, Washington Square Park, New York, NY 10003.
}

mitia, 1978; Descarries et al., 1982), little ultrastructural evidence exists for serotonergic axons having an association with these glia cells or their processes.

Ascending 5-HT-containing fibers, first visualized with histochemical fluorescence methods, were classically described as being unmyelinated (Dahlstrom and Fuxe, 1965; Fuxe, 1965). These workers noted that the 5-HT fibers were of two general types: either very fine and varicose or large and smooth. Light microscope immunocytochemical studies have confirmed that 5-HT fibers are not homogeneous. Lidov et al. (1980) described large axons in the medial forebrain bundle (MFB), diagonal band, and supracallosal stria and very fine axons in the cortical layer $(0.1$ to $0.5 \mu \mathrm{m}$ in diameter). Steinbusch (1981) described three fiber types: (1) long and fine fibers without any or with only a few varicosities $(0.2$ to $0.5 \mu \mathrm{m}),(2)$ thinner fibers with varicosities, and (3) fibers with varicosities without clear intervaricose connections. Finally, Kohler et al. (1981) described four fiber types, one of which they stated was large and possibly myelinated. 
Radioautographic and degeneration ultrastructural studies in various forebrain areas, such as caudate (Calas et al., 1976), cerebral ventricles (Richards et al., 1973), dorsomedial hypothalamus (Beaudet and Descarries, 1979), median eminence (Calas et al., 1974), neocortex (Descarries et al., 1975), substantia nigra (Parizek et al., 1971), and suprachiasmatic nucleus (Aghajanian et al., 1969), did not demonstrate the presence of myelinated serotonergic axons. Electrophysiological studies in the rat are generally consistent with 5-HT fibers being unmyelinated. Electrical stimulation of the midbrain raphe nuclei produces an inhibitory response in the hippocampus with a slow conduction velocity (Segal, 1975; Winson, 1980). The retrograde conduction velocities from the amygdala (Wang and Aghajanian, 1977) and from the hippocampus (Crunelli and Segal, 1982) to the raphe nuclei are also predominantly slow. The morphological and functional evidence for unmyelinated 5-HT axons supports a slow-acting and diffuse role for the serotonin system within the forebrain of vertebrates.

This generally accepted view requires re-examination. Electron microscope radioautographic analysis of the spinal cord of the rat (Ruda and Gobel, 1980) and the lower brainstem of the monkey (Chan-Palay, 1978a, b) has shown examples of myelinated fibers labeled after in vivo administration of $\left[{ }^{3} \mathrm{H}\right] 5-\mathrm{H}$ T . Also, recent electrophysiological studies have provided evidence for a fast conduction response component in both anterograde (Srebro et al., 1982) and retrograde (Crunelli and Segal, 1982) stimulation studies of the midbrain raphe nuclei. We have undertaken a quantitative analysis of the 5HT-immunoreactive (IR) axons in the medial forebrain bundle of the rat and monkey in order to answer two specific questions. One, are unmyelinated 5 -HT-IR fibers in contact with the myelin sheaths of nonserotonergic myelinated fibers? Two, are some of the 5-HT-IR fibers myelinated? We report here that more than half of the 5-HT-IR fibers in both the rat and monkey MFB are unmyelinated and make contact with nonserotonergic myelinated fibers. Myelinated 5-HT-IR axons were seen in both the rat and the monkey but were more frequent in the monkey.

\section{Materials and Methods}

Rats (Sprague-Dawley, female, Zivic Miller Breeders, $220 \mathrm{gm}$ ) and monkeys (Macaca fascicularis, female, Charles River Breeding Laboratory, $3.3 \mathrm{~kg}$ ) were pretreated with pargyline and tryptophan (rats, $200 \mathrm{mg} / \mathrm{kg}$; monkeys, $50 \mathrm{mg} / \mathrm{kg}$ ). The pargyline (Sigma; $50 \mathrm{mg} / \mathrm{ml}$ of saline) was injected intraperitoneally $30 \mathrm{~min}$ before an intraperitoneal injection of L-tryptophan (Sigma; $50 \mathrm{mg} /$ $\mathrm{ml}$ of saline). One hour later the animals were perfused through the ascending aorta with $4 \%$ paraformaldehyde, $0.2 \%$ glutaraldehyde, and $0.1 \% \mathrm{MgSO}_{4}$ in $0.1 \mathrm{M}$ phosphate buffer $(\mathrm{pH} 7.4)$ at $20^{\circ} \mathrm{C}$ for $10 \mathrm{~min}$ and continued with the same solution without the glutaraldehyde (total perfusion volume was $250 \mathrm{ml}$ in the rat and $1500 \mathrm{ml}$ in the monkey) for an additional $20 \mathrm{~min}$. The brains were postfixed at $5^{\circ} \mathrm{C}$ for at least $4 \mathrm{hr}$ (no glutaraldehyde) before being processed for immunocytochemistry. Twenty-micrometer sections of the lateral hypothalamus were cut on a Vibratome (Oxford). The primary anti- serum and the secondary sera were diluted in $0.1 \mathrm{M}$ Trisbuffered ( $\mathrm{pH} 7.4$ ) saline $(0.85 \%)$ containing $1 \%$ normal sheep serum and $0.1 \%$ Triton $\mathrm{X}-100$. The sections were incubated in the following sequence: (1) $18 \mathrm{hr}$ at $5^{\circ} \mathrm{C}$ followed by $2 \mathrm{hr}$ at room temperature (RT) in antiserotonin serum at a dilution of $1 / 1500 ;(2) 30 \mathrm{~min}$ at RT in sheep anti-rabbit serum (1/100, Antibodies Inc.); (3) $1 \mathrm{hr}$ at RT in peroxidase-antiperoxidase complex (1/ 100, Miles Laboratories); (4) $10 \mathrm{~min}$ at RT in $0.05 \% 3,3-$ diaminobenzidine containing $0.2 \%$ nickel ammonium sulfate in $0.1 \mathrm{M}$ Tris-buffered ( $\mathrm{pH} 7.4$ ) saline (Azmitia and Gannon, 1982), (5) 5 to $10 \mathrm{~min}$ at RT in the same solution as (4) with the addition of $0.003 \%$ hydrogen peroxide. The sections were subsequently postfixed for 1 $\mathrm{hr}$ at $20^{\circ} \mathrm{C}$ in $2 \%$ osmium tetroxide containing $1.5 \%$ potassium ferricyanide in $0.1 \mathrm{M}$ phosphate buffer $(\mathrm{pH}$ 7.2) and then block stained in $0.5 \%$ uranyl acetate at $5^{\circ} \mathrm{C}$ for $30 \mathrm{~min}$. Ultrathin sections were taken from the surface of Epon/Araldite-embedded tissue slices and viewed on the electron microscope without further heavy metal staining.

The specificity of the antibody used against serotonin has been demonstrated by a lack of staining following in vitro immunoabsorption with serotonin or following the in vivo injection of specific neurotoxins. Furthermore, there was no staining in brain areas known not to have serotonergic neurons. Lauder and co-workers (1982) showed that the staining with this antibody was completely eliminated by preabsorption with $5-\mathrm{HT}$ at a concentration of $500 \mu \mathrm{M}$ but not with dopamine or noradrenaline at a concentration of $4000 \mu \mathrm{M}$. Frankfurt et al. (1981) showed complementary results in the raphe nuclei and substantia nigra of the rat using millimolar concentrations of 5-HT, dopamine, and norepinephrine. Furthermore, in this latter study positive cellular staining was confined to the known serotonergic nuclei and a single group of cells in the dorsomedial hypothalamus. Frankfurt and Azmitia (1983) have shown that microinjection directly into the rat MFB of 5,7-dihydroxytryptamine, but not 6 -hydroxydopamine (both at $3 \mu \mathrm{g} / 0.3$ $\mu \mathrm{l})$, eliminated the 5 -HT-IR fibers by 12 days postlesion.

\section{Results}

Light microscopic analysis of the rat and monkey hypothalamus was used to locate the position of the MFB (Fig. 1). In the rat this fiber tract contained the majority of ascending 5-HT-IR fibers, whereas in the monkey the major ascending pathway appeared to be the dorsal raphe cortical tract (DRCT). The DRCT is situated dorsal to the MFB and just medial to the internal capsule. The analysis described in this report considers only those 5-HT-IR fibers confined to the MFB.

5-HT-IR unmyelinated fibers were seen throughout the MFB of both the rat (Fig. 2) and the monkey (Fig. 3). The labeled axons were usually seen as individual fibers surrounded by unlabeled processes. Occasionally, paired 5-H'T-IR fibers were observed (Fig. 3C) in both the monkey and rat MFB. Specific intraneuronal labeling was detected within small vesicles and the tubular profiles of the smooth endoplasmic reticulum (SER). These two sites comprise the membrane-enclosed compartments of the 5-HT-IR. Evidence of 5-HT immunoreac- 

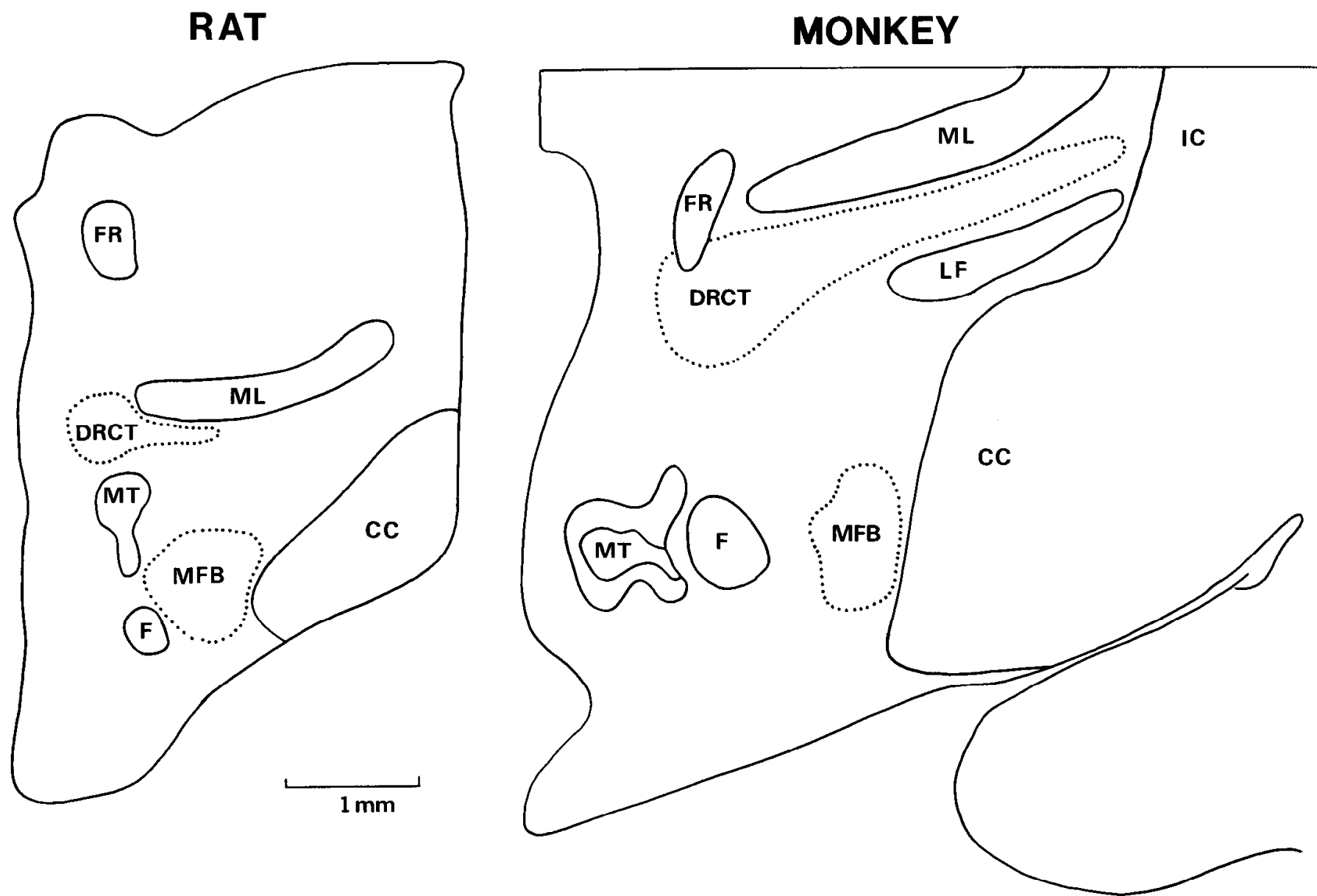

Figure 1. Schematic drawing of coronal sections at the level of the posterior hypothalamus in the rat and monkey brain. The two main ascending pathways are the medial forebrain bundle $(M F B)$ and the dorsal raphe cortical tract $(D R C T)$ and these are outlined with dashed lines. The relative size and position of the MFB is similar in these two species. However, the DRCT is much larger in the monkey than in the rat brain, although it does occupy a similar relative position in both species. $C C$, crus cerebri; $F$, fornix; $F R$, fasciculus retroflexus; $I C$, internal capsule; $M L$, medial lemniscus; $M T$, mammillothalamic tract.

tivity was also found on the outer membranes of mitochondria and in patches along the inner face of the axolemma. These sites of 5-HT immunoreactivity represent the membrane-attached compartment.

In both species, more than half of the unmyelinated fibers immunoreactive for 5 -HT were in contact with myelinated fibers: $55 \%$ (817 contacts of 1481 labeled profiles) in the rats $(n=3)$ and $54 \%$ (394 contacts of 736 labeled profiles) in the monkeys $(n=2)$. The contact sites were characterized by a close apposition of the 5HT-IR axon to the outer myelin membrane (Figs. $2 C$ and $3 C$ ).

Myelinated 5-HT-IR fibers were seen in both rats (Fig. $2 B$ ) and monkeys (Fig. $3 B$ ). The percentage of labeled myelinated fibers to the total number of labeled fibers was greater in the monkey $(25 \%, 223$ of 956 profiles) than in the rat MFB (0.7\%, 10 of 1491 profiles). The cellular distribution of reaction product was similar to that of unmyelinated fibers but usually denser (Figs. $2 B$ and $3 B$ ).

Unmyelinated fibers varied in their diameter between 0.2 and $1.25 \mu \mathrm{m}$, whereas the myelinated 5-HT-IR axons were generally larger, having diameters between 1.0 and $2.1 \mu \mathrm{m}$.

\section{Discussion}

The analysis presented in this study was confined to the MFB of the rat and monkey. In the rat, the majority of ascending serotonergic fibers travel within this pathway, although additional routes have been observed using histochemical fluorescence (Seiger and Olson, 1973) and anterograde tracing methods (Azmitia and Segal, 1978). In this latter study, a group of fibers was seen just dorsal to the MFB which could be followed to the cerebral cortex via the internal capsule. This DRCT had been seen previously using histochemical fluorescence but was assumed to join the MFB in more anterior sections (Fuxe and Jonsson, 1974). In the monkey, we observed a very large number of fibers in a position analogous to the DRCT of the rat. This route to the cerebral cortex may in fact contain more ascending 5-HT fibers than does the MFB in the monkey. Thus, future studies of the ascending serotonergic fibers in the primate brain should include those fibers lying outside the boundaries of the MFB.

Caution should be exerted in the interpretation of the subcellular distribution of the 5-HT immunoreactivity because of possible displacement of the antigen $\left(5-\mathrm{H}^{\mathrm{T}} \mathrm{T}\right)$ 

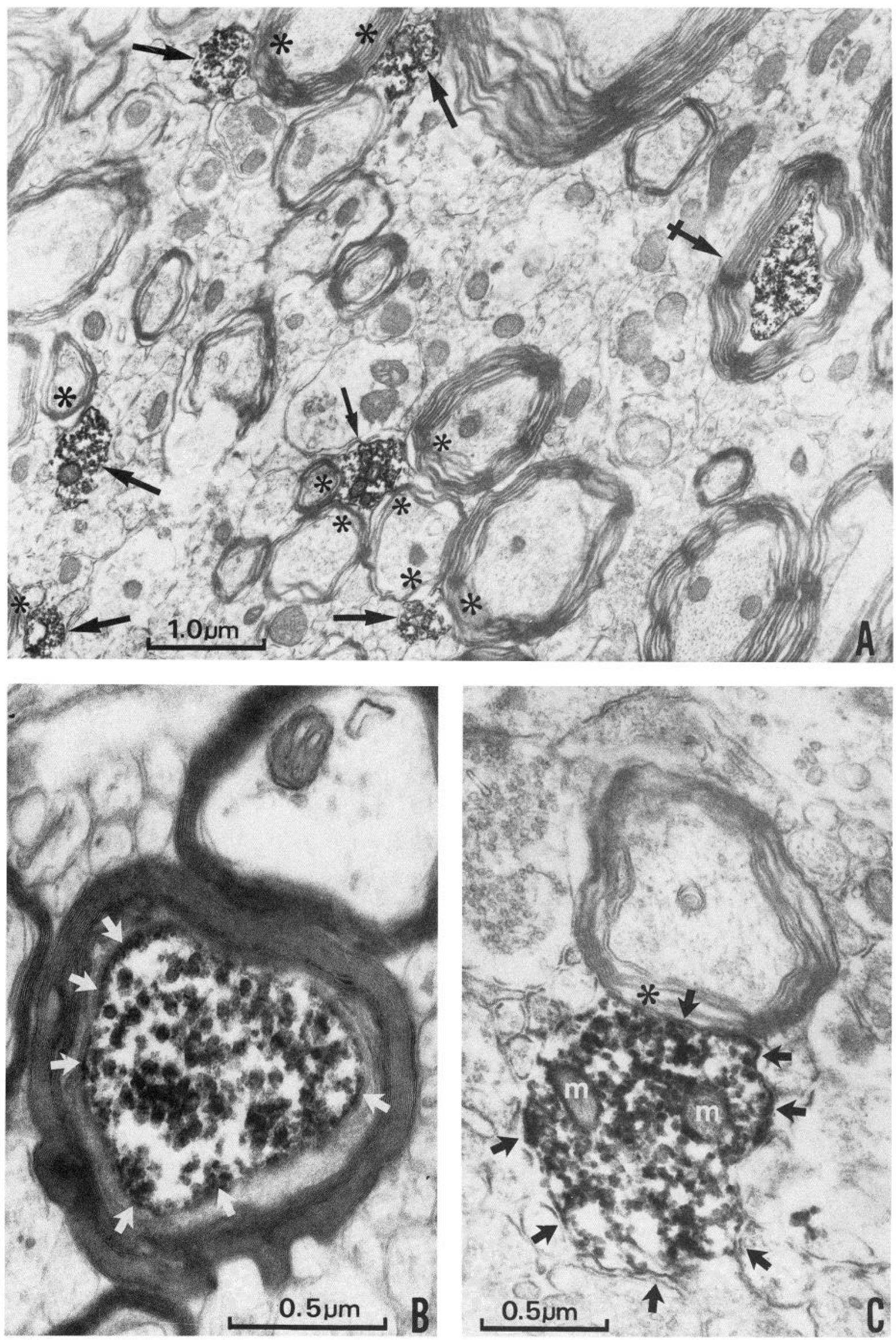
during fixation and the immunocytochemical procedures. Nevertheless, our results obtained in the MFB are consistent with previous immunocytochemical and radioautographic reports. In our report, the 5-HT immunoreactivity was detected in both membrane-enclosed and membrane-bound compartments. The membrane-enclosed compartment consisted of 5-H'T immunoreactivity within small clear vesicles ( 30 to $35 \mathrm{~nm}$ in diameter) and the tubular profiles of SER of labeled axons. Lighter staining appeared within microtubules and large vesicles (65 to $70 \mathrm{~nm}$ in diameter). The reaction product in the other compartment was detected on the outer mitochondrial membrane, on the periphery of the large dense core vesicles, and on the inner face of the axolemma. Pasik et al. (1982), in the monkey caudate, reported 5-HT immunoreactivity in vesicles, on the outer mitochondrial membrane, and associated with microtubules. Maley and Elde (1982), working in the cat nucleus of the solitary tract, reported 5-HT immunoreactivity associated with large granular vesicles ( 80 to $150 \mathrm{~nm}$ in diameter) in fibers and with small vesicles in terminals. This localization supported the hypothesis, originally proposed by Chan-Palay (1976), that 5-HT is transported down the axon in large vesicles and transferred to small vesicles at the terminal. This interpretation is not consistent with our findings of ascending 5-HT-IR fibers in the MFB where the 5-HT immunoreactivity is associated with small vesicles and tubular profiles of the SER.

Intraventricular administration of $\left[{ }^{3} \mathrm{H}\right] 5-\mathrm{HT}$ has been localized by several groups using electron microscope radioautography. Chan-Palay (1978b) observed silver grains (SGs) overlying vesicles, SER, mitochondria, microtubules, and neural filaments. Gamrani and Calas (1980) reported that SGs in the raphe neurons were localized within mitochondria when monoamine oxidase was inhibited, but homogeneously distributed throughout the cytoplasm when it was not inhibited. Descarries and co-workers (1982), in a radioautographic study of the dorsal raphe nucleus, used statistical analysis of the SG labeling to demonstrate that the label was largely confined to dense bodies (lysosomes) and secondarily to mitochondria and the cytoplasmic membrane. Finally, Araneda and colleagues (1980) showed that after intracerebral injections of $\left[{ }^{3} \mathrm{H}\right] 5-\mathrm{HT}$, the SGs were concentrated over selected terminals and retrogradely transported to the raphe cell bodies inside mitochondria. Further studies not using perfusing procedures are required to establish the subcellular localization of the endogenous serotonin.

Oligodendrocytes in the CNS are divided into two major classes: fascicular and pericellular. The myelin sheaths in the brain are formed by a layering of membrane processes from the fascicular type, whereas the neuronal soma forms contacts with the soma of the pericellular type. Thus, a neuron may interact with oligodendrocytes from its cell body to its terminal branch. Previous work in the dorsal raphe nucleus demonstrated that a large number of the neurons were in contact with oligodendrocytes (pericellular type) (Azmitia, 1978; Descarries et al., 1982). We have extended this observation with immunocytochemical studies of the dorsal raphe nucleus to show that 5-HT-IR perikarya, axons, and dendrites form contacts with oligodendrocytes (unpublished observations). We now report that most of the 5HT-IR axons in the MFB of the rat and monkey make contact with unreactive myelinated axons. Future studies should investigate whether other unmyelinated fiber systems (e.g., dopaminergic, noradrenergic) share a similar relationship to myelinated fibers.

Ramon y Cajal believed that myelin-producing cells played a key role in guiding regenerating fibers in the peripheral nervous system. He wrote that "the process of attraction and penetration of the new fibers into the peripheral stump is closely related to the vitality of the cells of Schwann" (Ramon y Cajal, 1928, p. 339). In the CNS, serotonergic axons sprout following damage to their axons (regeneration; Bjorklund et al., 1973) or damage to neighboring homotypic axons (collateral sprouting; Azmitia et al., 1978). Although the specific sprouting signal is unknown, regenerating axons have been described as preferentially following myelinated pathways to reinnervate vacated sites in the forebrain (Bjorklund et al., 1973). In addition, transplanted fetal serotonergic neurons send their axons along myelinated pathways of the host adult brain to innervate selected terminal regions (Azmitia et al., 1981; Bjorklund et al., 1976). The frequent contacts seen between unmyelinated 5-HT-IR axons and the myelin sheath of nonserotonergic fibers may represent the anatomical substrate for these examples of directed growth.

As has been frequently shown, 5-HT-containing axons are unmyelinated in a variety of invertebrate species which include Aplysia (Weinreich et al., 1973), leech (Coggeshall, 1971), and lobster (Livingstone et al., 1981). Our study has shown that, in rats, these 5-HT axons are only rarely myelinated, whereas in a higher form of mammal, as represented by monkeys, nearly a quarter of the fibers are found to be myelinated. These observations suggest a progression of increased myelination in this primitive brainstem system from invertebrates to higher mammals. This progression of a chemically identified neuronal system supports the hypothesis of a generalized

Figure 2. Electron photomicrographs of the rat MFB showing 5-HT-immunoreactive axons in cross-section. A, A low power view showing six unmyelinated axons (arrows) and a single myelinated axon (arrow with bar) immunoreactive to a specific 5HT antibody. All immunoreactive axons shown are in close apposition to unreactive myelinated axons (asterisk). $B$, High power electron micrograph of myelinated axon stained with the 5-HT antibody. The staining is mainly restricted to vesicles and patches along the cytoplasmic leaf of the axonal membrane (arrow). $C$, unmyelinated axon shown in close apposition to unreactive myelinated axon (asterisk). The cytoplasmic distribution of the reaction product is as described above in $B$. In addition, the outer mitochondrial membrane is stained. Note the densities between the immunoreactive axon and the unreactive myelinated and unmyelinated fibers (arrows). 

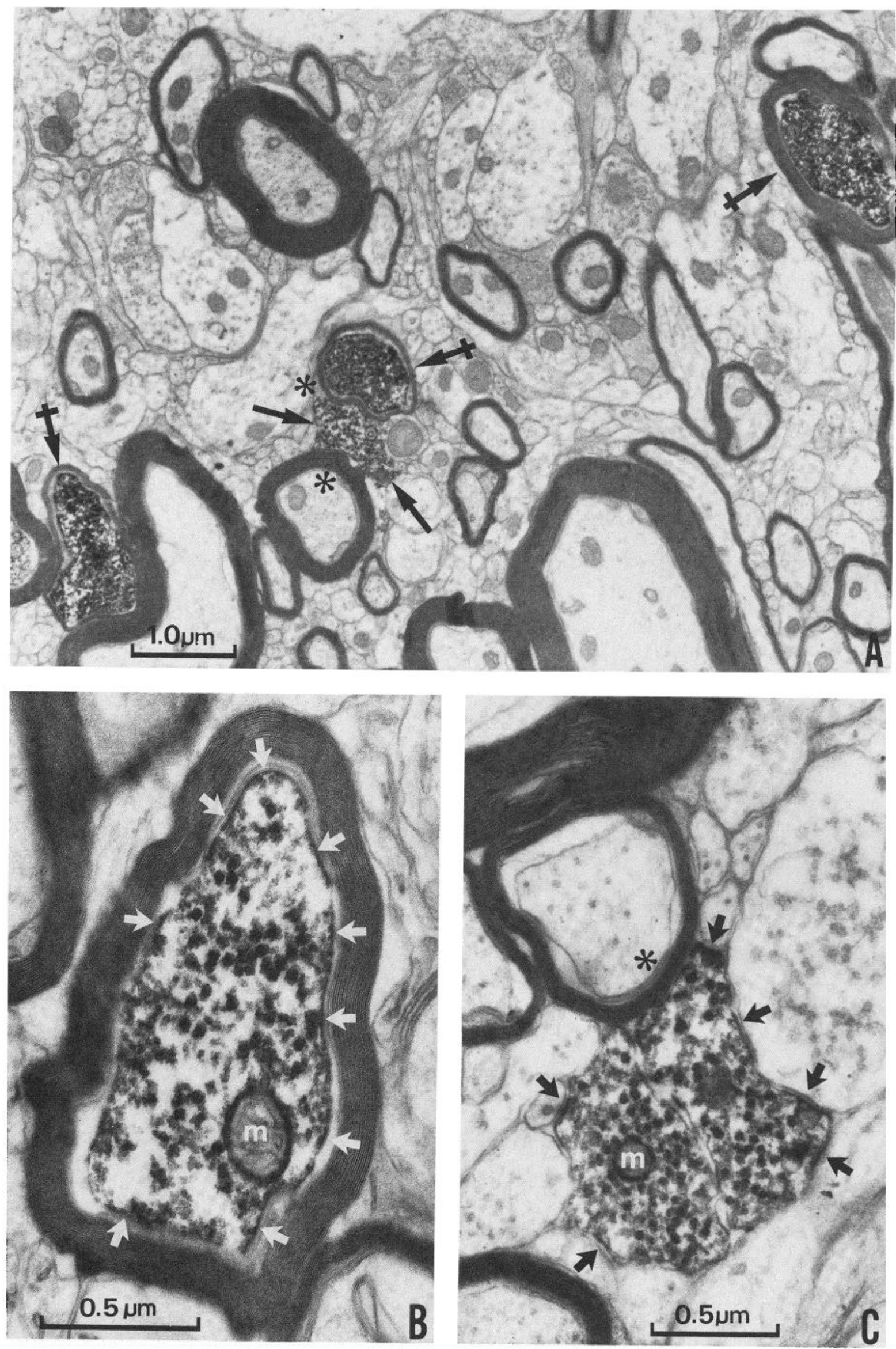
trend in increased myelination within evolution (Bishop and Smith, 1964; Sanides, 1970). The significance of this myelination trend to the function of serotonin in the human brain remains to be studied.

\section{References}

Aghajanian, G. K., F. E. Bloom, and M. H. Sheard (1969) Electron microscopy of degradation within the serotonin pathway of rat brain. Brain Res. 13: 266-273.

Araneda, S., H. Gamrani, C. Font, A. Calas, J. F. Pujol, and P. Bobillier (1980) Retrograde axonal transport following injection of $\left({ }^{3} \mathrm{H}\right)$-serotonin into the olfactory bulb. II. Radioautographic study. Brain Res. 196: 417-427.

Azmitia, E. C. (1978) The serotonin-producing neurons of the midbrain median and dorsal raphe nuclei. In Handbook of Psychopharmacology, L. L. Iversen, S. D. Iversen, and S. H. Snyder, eds., Vol. 9, pp. 122-314, Plenum Press, New York.

Azmitia, E. C., and P. J. Gannon (1982) Immuno-reactive myelinated 5-HT axons in the medial forebrain bundle of monkey and rat. Soc. Neurosci. Abstr. 8: 134.

Azmitia, E. C., and M. Segal (1978) An autoradiographic analysis of the differential projections of the dorsal and median raphe nuclei in the rat. J. Comp. Neurol. 179: 641-659.

Azmitia, E. C., A. M. Buchan, and J. H. Williams (1978) Structural and functional restoration by collateral sprouting of hippocampal 5-H'T axons. Nature 274: 374.

Azmitia, E. C., M. J. Perlow, M. J. Brennan, and J. M. Lauder (1981) Fetal raphe and hippocampal transplants into adult and aged $\mathrm{C} 57 \mathrm{BL} / 6 \mathrm{~N}$ mice: A preliminary immunocytochemical study. Brain Res. Bull. 7: 703-710.

Beaudet, A., and L. Descarries (1979) Radioautographic characterization of a serotonin-accumulating nerve cell group in the adult rat hypothalamus. Brain Res. 160: 231-243.

Bishop, G. H., and J. M. Smith (1964) The size of nerve fibers supplying cerebral cortex. Exp. Neurol. 9: 484-501.

Bjorklund, A., A. Nobin, and U. Stenevi (1973) Regeneration of central serotonin neurons after axonal degeneration induced by 5,6-dihydroxytryptamine. Brain Res. 50: 214-220.

Bjorklund, A., U. Stenevi, and N. -A. Svendgaard (1976) Growth of transplanted monoaminergic neurons into the adult hippocampus along the perforant path. Nature 262: 787-790.

Calas, A., G. Alonso, E. Arnauld, and J. D. Vincent (1974) Demonstration of indolaminergic fibers in the median eminence of the duck, rat and monkey. Nature (Lond.) 250: 241-243.

Calas, A., M. J. Beeson, C. Gaughy, G. Alonso, J. Glowinski, and A. Cheramy (1976) Radioautographic study of in vivo incorporation of ${ }^{3} \mathrm{H}$-monoamines in the cat caudate nuckeus: Identification of serotonergic fibers. Brain Res. 118: 1-13.

Chan-Palay, V. (1976) On the identification of $\mathrm{CAT}_{2}$ serotonin axons in the mammalian cerebellum. The roles of large granular, small, and granular alveolate vesicles in transmitter storage, discharge, and reuptake-A hypothesis. In 'SIF Cells-Structure and Function of the Small, Intensely Fluorescent Sympathetic Cells, O. Eranko, ed., pp. 227-249,
Department of Health, Education and Welfare, Washington, D. C.

Chan-Palay, V. (1978a) The paratrigeminal nucleus. II. Identification and inter-relations of catecholamine axons, indolamine axons, and substance $\mathrm{P}$ immunoreactive cells in the neuropil. J. Neurocytol. 7: 419-442.

Chan-Palay, V. (1978b) Morphological correlates for transmitter synthesis, transport, release, uptake and catabolism: A study of serotonin neurons in the nucleus paragigantorcellularis lateralis. In Amino Acids as Chemical Transmitters, F. Fonnum, ed., pp. 1-30, Plenum Press, New York.

Coggeshall, R. E. (1971) Autoradiographic and chemical localization of 5-hydroxytryptamine in identified neurons in the leech. Anat. Rec. 172: 489-498.

Crunelli, V., and M. Segal (1982) Electrophysiological study of the rat nucleus raphe medianus. J. Physiol. (Lond.) 328: 43$44 \mathrm{P}$.

Dahlstrom, A., and K. Fuxe (1965) Evidence for the existence of monoamine neurons in the central nervous system. II. Experimentally induced changes in the intraneuronal amine levels of bulbospinal neuron systems. Acta Physiol. Scand. 64(Suppl 247): 1-36.

Descarries, L., A. Beaudet, and K. C. Watkins (1975) Serotonin nerve terminals in adult rat neocortex. Brain Res. 100:563588 .

Descarries, L., K. C. Watkins, S. Garcia, and A. Beaudet (1982) The serotonin neurons in nucleus raphe dorsalis of adult rat: A light and electron microscope radioautographic study. J. Comp. Neurol. 207: 239-254.

Frankfurt, M., and E. Azmitia (1983) The effect of intracerebral injections of 5,7-dihydroxytryptamine and 6-hydroxydopamine on the serotonin immunoreactive cell bodies and fibers in the adult rat hypothalamus. Brain Res. 261: 91-99.

Frankfurt, M., J. M. Lauder, and E. C. Azmitia (1981) The immunocytochemical localization of serotonergic neurons in the rat hypothalamus. Neurosci. Lett. 24: 227-232.

Fuxe, K. (1965) Distribution of monoamine nerve terminals in the central nervous system. Acta Physiol. Scand. 247: 37-85.

Fuxe, K., and G. Jonsson (1974) Further mapping of central 5 hydroxytryptamine neurons: Studies with Neurotoxic dihydroxytryptamine. Adv. Biochem. Psychopharmacol. 10:1-12.

Gamrani, H., and A. Calas (1980) Cytochemical, stereological and radioautographic studies of rat raphe neurons. Mikroskopie (Wien) 36: 1-11.

Kohler, C., V. Chan-Palay, and H. Steinbusch (1981) The distribution and orientation of serotonin fibers in the entorhinal and other retrohippocampal areas. Anat. Embryol. 161: $237-264$.

Lauder, J. M., and F. E. Bloom (1974) Ontogeny of monoamine neurons in the locus coeruleus, raphe nuclei and substantia nigra. I. Cell differentiation. J. Comp. Neurol. 155: 469-481.

Lauder, J. M., P. Petrusz, J. A. Wallace, A. DiNome, M. B. Wilkie, and K. McCarthy (1982) Combined serotonin immunocytochemistry and ${ }^{3} \mathrm{H}$-thymidine and autoradiography: In vivo and in vitro methods. J. Histochem. Cytochem. 30: 788-798.

Lidov, H. G. W., R. Grzanna, and M. E. Molliver (1980) The

Figure 3. Electron micrographs of monkey MFB showing 5-HT-immunoreactive axons in cross-section. A, A low power micrograph of a single unmyelinated axon (arrow) and three myelinated axons (arrows with bar). $B$, a high power electron micrograph showing a myelinated axon. Neurotubules appear to be only lightly stained. Specific staining is seen in small clear vesicles, the tubular profiles of smooth endoplasmic reticulum, the outer mitochondrial membranes, and in patches along the inner side of the axolemma (arrows). C, A pair of labeled unmyelinated axons shown in close apposition to an unreactive myelinated axon (asterisk). Immunoreactive densities in the stained axons are apposed both to myelinated and unmyelinated unreactive axons (arrows). The two immunoreactive fibers shown are separated by a glial process. 
serotonin innervation of the cerebral cortex in the rat: An immunohistochemical analysis. Neuroscience 5: 207-223.

Livingstone, M. S., S. F. Schaeffer, and E. A. Kravitz (1981) Biochemistry and ultrastructure of serotonergic nerve endings in the lobster: Serotonin and octopamine are contained in different nerve endings. J. Neurobiol. 12: 27-54.

Maley, B., and R. Elde (1982) The ultrastructural localization of serotonin immunoreactivity within the nucleus of the solitary tract of the cat. J. Neurosci. 2: 1499-1506.

Olson, L., and A. Seiger (1972) Early prenatal ontogeny of central monoamine neurons in the rat: Fluorescence histochemical observations. Z. Anat. Entwicklungs-gesch. 137: 301-316.

Parent, A. (1981) The anatomy of serotonin-containing neurons across phylogeny. In Serotonin Neurotransmission and Behavior, B. L. Jacobs and A. Gelperin, eds., pp. 3-34, MIT Press, Cambridge, MA.

Parizek, J., R. Hassler, and I. J. Bak (1971) Light and electron microscopic autoradiography of substantia nigra of rat after interventricular administration of tritium labelled norepinephrine, dopamine, serotonin and the precursors. Z. Zellforsch. 115: 137-148.

Pasik, P., T. Pasik, and J. Pecci-Saavedra (1982) Immunocytochemical localization of serotonin at the ultrastructural level. J. Histochem. Cytochem. 30: 760-764.

Ramon y Cajal, S. (1928) Experiments dealing with the transplantation of nerves or their products, designed to prove especially an attractive or neurotropic action on nerve sprouts. In Degeneration and Regeneration of the Nervous System, Vol. 1, Hafner Publishing Co., New York.

Richards, J. G., H. P. Lorez, and J. P. Tranzer (1973) Indolealkylamine nerve terminals in cerebral ventricles: Identification by electron microscopy and fluorescence hitochemistry. Brain Res. 57: 277-288.
Ruda, M. A., and S. Gobel (1980) Ultrastructural characterization of axonal endings in the substantia gelatinosa which take up $\left[{ }^{3} \mathrm{H}\right]$ serotonin. Brain Res. 184: 57-83.

Sanides, F. (1970) Functional architecture of motor and sensory cortices in primates in the light of a new concept of neocortex evolution. In The Primate Brain, C. R. Noback and W. Montagna, eds., pp. 137-208, Appleton-Century-Crofts, New York.

Segal, M. (1975) Physiological and pharmacological evidence for a serotonergic projection to the hippocampus. Brain Res. 94: 115-131.

Seiger, A., and L. Olson (1973) Late prenatal ontogeny of central monoamine neurons in the rat: Fluorescence histochemical observations. Z. Anat. Entwicklungs gesch. 140: 281-318.

Srebro, B., E. C. Azmitia, and J. Winson (1982) Effect of 5-HT depletion of the hippocampus on neuronal transmission from perforant path through dentate gyrus. Brain Res. 235: 142147.

Steinbusch, H. W. M. (1981) Distribution of serotonin immunoreactivity in the central nervous system of the rat-cell bodies and terminals. Neuroscience 6 : 557-618.

Wang, R. Y., and G. K. Aghajanian (1977) Inhibition of neurons in the amygdala by dorsal raphe stimulation: Mediation through a direct serotonergic pathway. Brain Res. 120: 85102.

Weinreich, D., M. W. McCaman, R. E. McCamam, and J. E. Vaughn (1973) Chemical, enzymatic and ultrastructural characterization of 5-hydroxytryptamine-containing neurons from the ganglia of Aplysia californica and Tritonia diomedia. J. Neurochem. 20: 969-976.

Winson, J. (1980) Raphe influences on neuronal transmission from perforant pathway through dentate gyrus. J. Neurophysiol. 44: 937-950. 